\title{
Delta sleep instability in children with chronic arthritis
}

\author{
M.C. Lopes ${ }^{1}$, C. Guilleminault ${ }^{3}$, A. Rosa ${ }^{4}$, C. Passarelli², S. Roizenblatt ${ }^{1}$ and S. Tufik ${ }^{1}$ \\ ${ }^{1}$ Departamento de Psicobiologia, ${ }^{2}$ Departamento de Pediatria, Escola Paulista de Medicina, Universidade \\ Federal de São Paulo, São Paulo, SP, Brasil \\ ${ }^{3}$ Sleep Disorders Clinic, Stanford University, Palo Alto, CA, USA \\ ${ }^{4}$ Evolutionary Systems and Biomedical Engineering Laboratory, Lisboa, Portugal
}

Correspondence to: M.C. Lopes, Rua Dr. Napoleão de Barros, 925, 04024-002 São Paulo, SP, Brasil

E-mail: m.cecilialopes@psicobio.epm.br

\begin{abstract}
The objective of the present study was to evaluate the expression of a cyclic alternating pattern (CAP) in slow wave sleep (SWS) in children with the well-defined chronic syndrome juvenile idiopathic arthritis (JIA). Twelve patients (9-17 years of age), 7 girls, with JIA were compared to matched controls by age, pubertal stage and gender. After one night of habituation in the sleep laboratory, sleep measurements were obtained by standard polysomnography with conventional sleep scoring and additional CAP analyses. The sleep parameters of the JIA and control groups were similar for sleep efficiency (91.1 $\pm 6.7 \mathrm{vs} 95.8 \pm 4.0)$, sleep stage in minutes: stage $1(16.8 \pm 8.5$ vs $17.8 \pm 4.0)$, stage $2(251.9 \pm 41$ vs $262.8 \pm 38.1)$, stage 3 (17.0 \pm 6.0 vs 15.1 \pm 5.7$)$, stage $4(61.0 \pm 21.7$ vs $77.1 \pm 20.4)$, and rapid eye movement sleep $(82.0 \pm 27.6$ vs $99.0 \pm 23.9)$, respectively. JIA patients presented nocturnal disrupted sleep, with an increase in short awakenings, but CAP analyses showed that sleep disruption was present even during SWS, showing an increase in the overall CAP rate $(P<0.01)$. Overall CAP rate during non-rapid eye movement sleep was significantly higher in pediatric patients who were in chronic pain. This is the first study of CAP in pediatric patients with chronic arthritis showing that CAP analyses can be a powerful tool for the investigation of disturbance of SWS in children, based on sleep EEG visual analysis.
\end{abstract}

Key words: Children; Chronic-arthritis; Cyclic alternating pattern; Sleep fragmentation

Presented at the XI Congresso Brasileiro do Sono, Fortaleza, CE, Brazil, November 11-14, 2007.

Research supported by Associação Fundos de Incentivo à Psicofarmacologia (AFIP). Publication supported by FAPESP.

Received November 5, 2007. Accepted October 22, 2008

\section{Introduction}

Pain has a strong influence on a person's quality of life with day-to-day fluctuations of well-being (1). Chronic painful physical conditions are an important cause of insomnia in patients (2), and it has been reported that pain is a major factor in the expression and evolution of depressive disorder (3). Changes in EEG sleep patterns of patients in pain, particularly because of rheumatoid arthritis, have been reported (4). These changes are expressed in sleep fragmentation, a high number of awakenings, reduction of sleep efficiency, increase in stage 1 non-rapid eye movement (NREM) sleep, with a consequent increase in daytime drowsiness (5), and in complaints of fatigue (6). It has been suggested that the presence of changes in sleep patterns is associated with an increase in severity of pain complaints (7). Despite the fact that investigation of sleep may be a useful tool in patients with chronic pain, polysomnography studies still remain controversial in understanding the influence of pain during sleep (8). Also, limited information exists on sleep and chronic pain in pediatric cases. The intrusion of alpha in slow wave sleep (SWS) called alpha-delta sleep has been described as an EEG pattern that can be seen in chronic painful physical conditions in adults (8-11). It can be demonstrated in subjects with fibromyalgia and rheumatoid arthritis $(6,12)$, but alpha-EEG sleep has also been reported in symptom-free subjects $(8,13)$. There is also controversy surrounding 
delta-alpha pattern: this is not a sensitive marker for the detection of chronic pain (8) and it is not noted in many fibromyalgic patients (14) or in patients with other chronic pain conditions $(8,15,16)$.

Compared with adults, children have different arousal responses to stimuli during all sleep stages (17). However, high rates of nocturnal awakenings and daytime sleepiness have been reported in children with juvenile idiopathic arthritis (JIA) $(18,19)$. There is no correlation between visual sleep scoring $(20,21)$ and sleep/wake disturbances in adults with rheumatoid arthritis (22). There is a clear need for a better understanding of sleep disruption caused by chronic pain not only in adults, but also in children where data are lacking.

The cyclic alternating pattern (CAP) is a sleep EEG pattern that has been linked to sleep instability of NREM sleep in adults with chronic pain. The aim of the present study was to investigate the presence of CAP in children with JIA. We hypothesized that there would be an increase in the CAP rate in SWS of children with JIA compared to healthy matched controls. We also compared the American Association of Sleep Medicine (AASM) arousal and conventional sleep parameters with CAP analyses.

\section{Subjects and Methods}

Twelve young individuals (7 girls, 9-17 years of age) with JIA and 12 healthy controls were prospectively recruited and evaluated during the active phase of the illness. These tests were conducted between 2000 and 2002. All patients met the criteria of the American College of Rheumatology for active polyarticular JIA (23) and were seen consecutively in a specialized clinic. The inclusion criteria were the presence of clinical activity of the illness and/or increased erythrocyte sedimentation rate and/or reactive C-protein for a minimum of 6 months indicating inflammation. Exclusion criteria were the presence of neuropsychiatric diseases, and the presence of clinical signs and symptoms of other sleep disorders. Control individuals were matched for age, gender and pubertal stage. They were recruited from the community and underwent clinical examination, answered sleep/wake questionnaires, and were submitted to a polysomnograph. In both groups, the participating individuals or their legal caretakers signed an informed consent and the protocol was approved by the Ethics Committee of São Paulo Federal University (UNIFESP). The participation of all subjects in this study was voluntary.

Recording and conventional analysis

All patients were followed by a pediatric rheumatologic clinician. After 1 week of taking sleep logs that were used to evaluate their sleep/wake schedule and normal sleep time, the patients were in a laboratory at 7:30 pm and underwent an adaptation-night to monitor their sleep. The following night examinations were carried out during usual sleep time with lights off based on sleep logs. The recordings had a minimum duration of $7.5 \mathrm{~h}$ of total sleep time, and were performed on a computerized sleep system (Stellate System $^{\mathrm{TM}}$, Harmonie 2.4, Canada) with a sampling frequency of $200 \mathrm{~Hz} /$ channel. Recordings included four channels for EEG (C3-A2, C4-A1, O1-A2, O2-A1), two for electrooculogram (right and left), two for electromyogram (chin and anterior tibial), one for electrocardiogram (modified V2 lead), and channels for oral and nasal thermistors, microphone, thoracic, abdominal belts and pulse oximetry (Ohmeda ${ }^{\mathrm{TM}}$, Finland). Evening and morning questionnaires were administered to assess subjective quality of wake and sleep prior to the study in the laboratory. After all recordings were collected, they were converted into European Data format (EDF) to reanalyze the signals into the Somnologica program.

Sleep stages and wakefulness were scored in 30-s epochs, according to standard criteria (20). The sleep parameters analyzed were: sleep onset latency defined as three consecutive epochs of stage 1 ; total sleep time (TST); sleep efficiency (TST/total recording time); awake time after sleep onset. The NREM and rapid eye movement (REM) sleep stages and percent of TST were recorded. Arousals were calculated with identification of short ( $\geq 3 \mathrm{~s}$ ) arousal according to the AASM definition (21). Arousals were scored based on the definition for adults that does not include delta activity in each event. Arousal was defined as an abrupt EEG shift toward fast activity such as $8-13 \mathrm{~Hz}$ (alpha) or $>16 \mathrm{~Hz}$ (beta). In REM sleep, an increase in amplitude of submental EMG was required to score an arousal event. A minimum interval of $10 \mathrm{~s}$ of continuous sleep was needed to score each event. An arousal index was derived from these tabulations.

\section{CAP visual analysis during NREM sleep}

CAP parameters were detected visually according to the CAP Consensus Report (22). Two investigators independently scored either sleep/wake architecture based upon the criteria of Rechtschaffen and Kales (20) and CAP analyses were according to international criteria (22). These investigators were blind to clinical information. CAP phase $A$ is considered to be periodic EEG activity during NREM sleep with an activation phase lasting 2-60 s, which may include high-voltage slow waves (synchronization) or lowvoltage fast waves (desynchronization). CAP phase $B$ is described as the interval between two $A$ phases, with a 
duration of 2-60 s, corresponding to stage-related background activity. CAP cycles were defined as the sum of $A$ and $B$ phases. CAP sequences, identified by repetitive clusters of stereotyped EEG features, were analyzed for at least two consecutive CAP cycles (see Table 1).

The CAP parameters analyzed during NREM sleep were: CAP rate (percent of CAP sequences in total NREM sleep), CAP time, CAP cycle count and duration, CAP phase $A$ count and duration, CAP phase $B$ count and duration, and CAP sequence count and duration. Phase $A$ was divided into three subtypes: subtype A1 with predominance of synchronized EEG activity, and less than $20 \%$ desynchronization, such as delta bursts, $\mathrm{K}$ complex sequences, vertex waves, and polyphasic bursts (of slow and fast rhythms); subtype $A 2$, scored in the presence of 20 to $50 \%$ desynchronized EEG activity, with predominance of polyphasic bursts; subtype $A 3$, at least $50 \%$ of the EEG activity comprised low amplitude fast rhythms, such as K-alpha complexes, AASM arousals, and polyphasic bursts. Phase A subtype indexes were calculated and reported as the percent of phase $A 1, A 2$, or $A 3$ per hour of NREM sleep.

\section{Statistical analysis}

Central tendency measures were reported as means and standard deviation. The $t$-test for independent samples was used for identifying differences in sleep parameters, with a significance level set at $P<0.05$. One-way ANOVA, followed by the Tukey test, was used to determine the statistically significant differences between CAP parameters during NREM sleep stages in the JIA group. The level of significance for analysis of variance was set at $P<0.01$. After determining the normality of distribution, correlations were obtained between conventional sleep parameters and CAP parameters, as well as between CAP events and AASM arousals with Spearman's correlation coefficient $\left(r_{S}\right)$. The level of significance for the correlation tests was set at $\mathrm{P} \leq 0.05$.

\section{Results}

A description of the participants, patients and controls, is presented in Table 2. Sleep quality was assessed by questions evaluating sleep satisfaction. All children and adolescents reported that the night's sleep in the sleep

Table 1. Scoring system for sleep patterns.

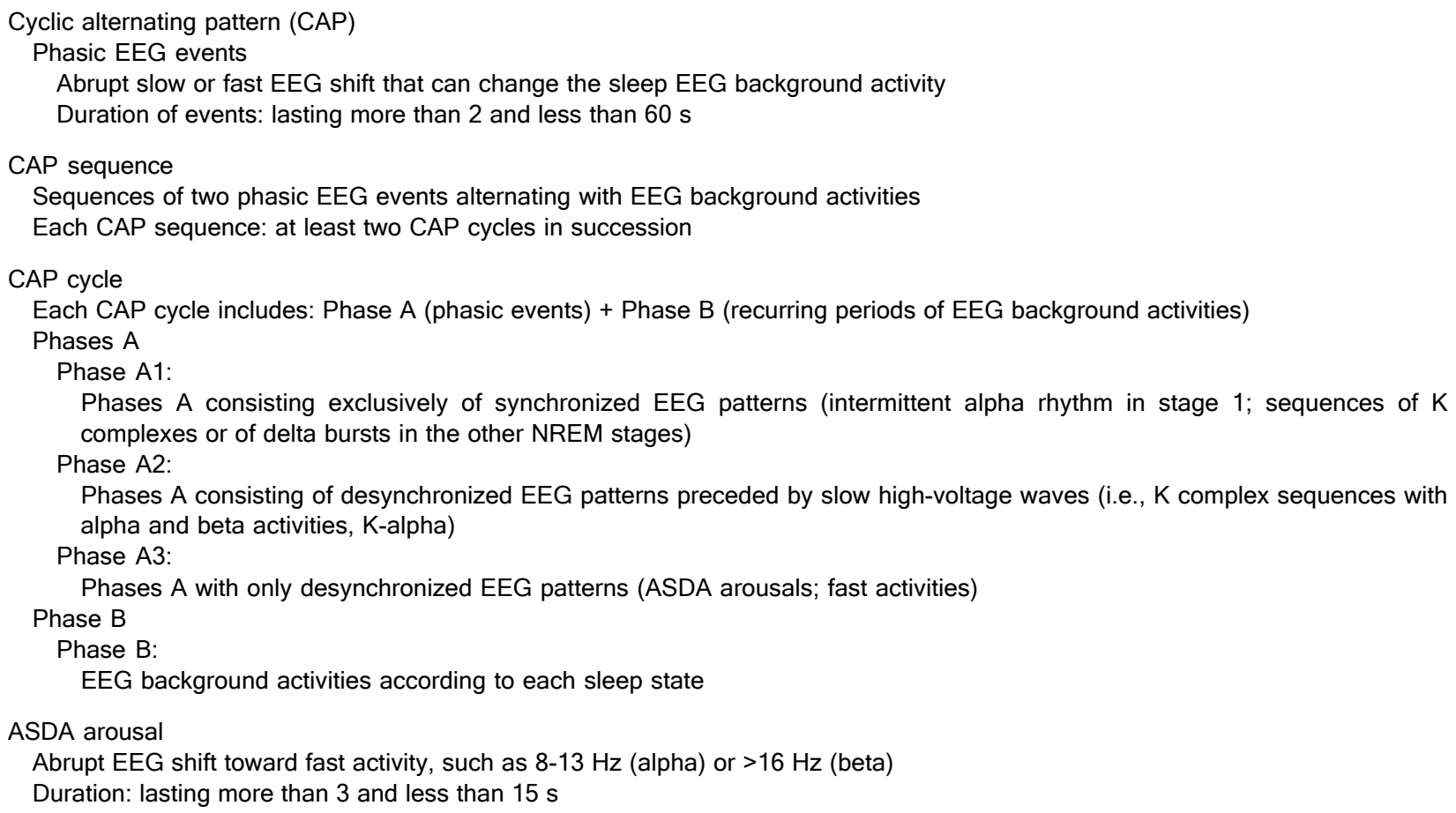

The scoring system was taken from Refs. 20 and 21. NREM = non-rapid eye movement sleep; ASDA = American Sleep Disorders Association. 
laboratory was no different from sleep at home. The patients had complaints about their sleep due to pain for at least 2 months prior to the sleep recording. The results of the nocturnal sleep analysis are presented in Table 3. JIA patients had significantly more arousals than controls. They also presented less time in stage 4 NREM sleep, had longer total sleep time, and wake time after sleep onset (WASO; P $<0.05)$. The fact that no significant changes in sleep stages were noted is a reflection of a scoring artifact, because short arousals $<10 \mathrm{~s}$ do not lead to a change in epoch scoring when using Rechtschaffen and Kales international scoring scale (20). Sleep latency, sleep efficiency, stages 1-3 NREM,

Table 2. Characteristics of the participants of this study.

\begin{tabular}{lcc}
\hline & JIA $(\mathrm{N}=12)$ & Controls $(\mathrm{N}=12)$ \\
\hline Age (years) & $12.5 \pm 2.4$ & $13.1 \pm 2.0$ \\
Girls:Boys & $7: 5$ & $7: 5$ \\
$\quad \begin{array}{l}\text { Time of disease } \\
\quad>3 \text { years }\end{array}$ & 7 & - \\
$\quad<3$ years & 5 & - \\
Impaired joints $(\mathrm{N})$ & $11.7 \pm 11$ & 0 \\
$\quad$ Erythrocyte sedimentation & $37.6 \pm 27.4$ & Not detected \\
$\quad$ rate $(\mathrm{mm} / \mathrm{h})$ & &
\end{tabular}

Data are reported as means \pm SD. JIA $=$ juvenile idiopathic arthritis; $N=$ number of subjects. There were no significant differences between age or gender ratios ( $P>0.05$, U-test).

Table 3. Sleep parameters.

\begin{tabular}{lcc}
\hline & JIA $(\mathrm{N}=12)$ & Controls $(\mathrm{N}=12)$ \\
\hline Sleep latency (min) & $17.6 \pm 15.4$ & $12.1 \pm 7.2$ \\
Total sleep time (min) & $477.1 \pm 43.1^{*}$ & $429.5 \pm 51.9$ \\
WASO (min) & $43.1 \pm 2.7^{*}$ & $19.8 \pm 5.0$ \\
Sleep efficiency (\%) & $91.1 \pm 6.7$ & $95.8 \pm 4.0$ \\
Stage 1 NREM (min) & $16.8 \pm 8.5$ & $17.8 \pm 10.4$ \\
Stage 2 NREM (min) & $251.9 \pm 41.0$ & $262.8 \pm 38.1$ \\
Stage 3 NREM (min) & $17.0 \pm 6.0$ & $15.1 \pm 5.7$ \\
Stage 4 NREM (min) & $61.0 \pm 21.7^{*}$ & $77.1 \pm 20.4$ \\
REM (min) & $82.0 \pm 27.6$ & $99.0 \pm 23.9$ \\
NA-NREM sleep & $69.9 \pm 28.6^{*}$ & $39.2 \pm 17.4$ \\
NA-REM sleep & $12.9 \pm 9.2$ & $9.9 \pm 6.7$ \\
Arousal index & $11.2 \pm 5.2^{*}$ & $5.5 \pm 2.4$ \\
Arousal index in REM sleep & $9.4 \pm 5.4^{*}$ & $4.8 \pm 2.1$ \\
Arousal index in NREM sleep & $8.4 \pm 3.6^{*}$ & $4.7 \pm 1.7$ \\
\hline
\end{tabular}

Data are reported as means $\pm \mathrm{SD}$. JIA $=$ juvenile idiopathic arthritis; WASO = wake time after sleep onset; NREM and REM = non-rapid and rapid eye moviment sleep; NA-NREM sleep = number of ASDA arousals in non-rapid eye movement sleep; NA-REM sleep = number of ASDA arousals in rapid eye movement sleep. $\mathrm{ASDA}=$ American Sleep Disorders Association. ${ }^{*} \mathrm{P}<$ 0.05 compared to controls (U-test).
REM, and number of arousals in REM sleep were not significantly different between groups. In fact, children with JIA showed more sleep fragmentation scored as short arousals than healthy controls.

CAP analyses showed several significant differences $(P<0.05$, $t$-test; see Table 4$)$. The percentage of CAP in stage 4 in JIA patients was significantly higher than in control subjects $(93.6 \pm 6.3$ vs $81.5 \pm 14.8 \%$, respectively; $\mathrm{P}<0.01, t$-test). In the patient group, the CAP parameters during stages of NREM sleep (Table 5) were increased in stages 3 and 4 , and the duration of phase $A$ was higher in stage 4 than in stage $2(P<0.01$, ANOVA; $P<0.01$, post hoc Tukey test). All CAP phase $A$ indexes were significantly different (Table 4). The number of CAP-A2 subtypes was higher in stage 3 of NREM sleep, in the patient group than in the control group $(P<0.01, t$-test $)$.

Several significant correlations were detected in the parameters of JIA patient group using the Spearman test. A positive correlation was observed between the number of EEG arousals and the sum of phases $A 2$ and $A 3$ subtypes $\left(r_{S}=0.80 ; P<0.01\right)$, but not with phase A1 subtype $\left(r_{S}=-0.08 ; P=0.73\right)$. A positive correlation was found between duration in minutes of stage 1 and CAP rate $\left(r_{S}=\right.$ 0.47 ; $P$ <.01), but not with AASM arousal index in NREM sleep $\left(r_{S}=-0.20 ; P=0.83\right)$. A positive correlation was also found between WASO and the index of phase A2 subtype during SWS $\left(r_{S}=0.62 ; P<0.01\right)$. A positive correlation was also found between the number of $A 3$ subtype events and sleep stage 2 duration $\left(r_{S}=0.72 ; P<0.01\right)$, but this was not statistically significant for AASM arousal $\left(r_{S}=-0.15 ; P=\right.$ 0.90 ). The number of joints with impairment was positively correlated with CAP rate $\left(r_{S}=0.24 ; P<0.01\right)$ and AASM arousal index $\left(r_{S}=0.29 ; P<0.01\right)$, but not with sleep

Table 4. Cyclic alternating pattern (CAP) parameters during nonrapid eye movement (NREM) sleep.

\begin{tabular}{lcc}
\hline & JIA $(\mathrm{N}=12)$ & Controls $(\mathrm{N}=12)$ \\
\hline CAP rate (\%) & $66.0 \pm 2.7^{*}$ & $52.7 \pm 8.6$ \\
CAP time (min) & $227.2 \pm 30.2^{*}$ & $195.7 \pm 29.6$ \\
CAP cycle number & $538.9 \pm 95.7^{*}$ & $446.3 \pm 96.7$ \\
Duration cycle (s) & $25.5 \pm 2.3$ & $26.8 \pm 2.8$ \\
Duration A phase (s) & $8.6 \pm 1.3$ & $8.2 \pm 1.2$ \\
Duration B phase (s) & $17.0 \pm 1.6^{*}$ & $18.6 \pm 2.1$ \\
CAP sequence duration (s) & $484.3 \pm 87.3^{*}$ & $391.7 \pm 131.4$ \\
Index of phase A subtypes & & \\
A1 & $60.9 \pm 9.2^{*}$ & $81.5 \pm 9.1$ \\
A2 & $25.3 \pm 4.6^{*}$ & $13.3 \pm 7.8$ \\
A3 & $13.0 \pm 6.5^{*}$ & $5.7 \pm 2.5$ \\
\hline
\end{tabular}

Data are reported as means $\pm \mathrm{SD}$. JIA = juvenile idiopathic arthritis. ${ }^{*} \mathrm{P}<0.01$ compared to controls (U-test). 
Table 5. Cyclic alternating pattern (CAP) expression during non-rapid eye movement sleep stages in patients with juvenile idiopathic arthritis.

\begin{tabular}{|c|c|c|c|c|c|c|}
\hline & Stage 1 & Stage 2 & Stage 3 & Stage 4 & ANOVA F $(3,27)$ & Significant post hoc \\
\hline CAP rate (\%) & $26.6 \pm 11.5$ & $58.4 \pm 6.3$ & $90.8 \pm 9.7$ & $93.6 \pm 6.3$ & 95.7 & $\mathrm{~S} 4, \mathrm{~S} 3>\mathrm{S} 2, \mathrm{~S} 1^{*}$ \\
\hline CAP time (min) & $5.4 \pm 3.8$ & $144.1 \pm 39.0$ & $20.7 \pm 18.7$ & $57.1 \pm 20.9$ & 71.6 & $\mathrm{~S} 2>\mathrm{S} 4>\mathrm{S} 3, \mathrm{~S} 1^{*}$ \\
\hline CAP cycles $(\mathrm{N})$ & $10.4 \pm 7.1$ & $334.3 \pm 99.0$ & $54.5 \pm 49.9$ & $139.3 \pm 54.9$ & 50.5 & $\mathrm{~S} 2>\mathrm{S} 4>\mathrm{S} 3, \mathrm{~S} 1^{*}$ \\
\hline CAP cycles (s) & $29.8 \pm 5.7$ & $26.1 \pm 2.4$ & $23.6 \pm 4.0$ & $25.0 \pm 2.8$ & 5.1 & $\mathrm{~S} 1>\mathrm{S} 3, \mathrm{~S} 4^{*}$ \\
\hline Phase A (s) & $8.5 \pm 3.1$ & $7.8 \pm 1.4$ & $9.5 \pm 1.1$ & $10.2 \pm 1.7$ & 19.4 & $S 4>S 2^{*}$ \\
\hline Phase B (s) & $21.4 \pm 5.3$ & $18.3 \pm 1.5$ & $14.5 \pm 3.7$ & $14.7 \pm 2.3$ & 9.9 & S1>S3,S4; S2>S3* \\
\hline
\end{tabular}

Data are reported as means $\pm \mathrm{SD}$. ${ }^{*} \mathrm{P}<0.05$ (post hoc comparison Tukey test).

parameters scored using Rechtschaffen and Kales criteria. Finally, the erythrocyte sedimentation rate was correlated with WASO in minutes $\left(r_{S}=0.76 ; P<0.01\right)$ and negatively correlated with CAP rate $\left(r_{S}=-0.48 ; P<0.01\right)$, but not correlated with the AASM arousal scores.

\section{Discussion}

This study shows the potential value of CAP sleep analysis of child patients with chronic pain. All of these patients reported sleep abnormalities. However, there were no significant changes in the time spent in each sleep stage when comparing patients with controls, and the total sleep time was even longer in patients compared with controls. Significant changes were seen only when short arousals were tabulated. The investigation of CAP indicated an abnormal instability of NREM sleep in JIA subjects shown by a significant increase in CAP rate, particularly in delta sleep. The increase in CAP rate has also been found in healthy subjects during the peripubertal period (24), in children with chronic snoring (23), in children with chronic sleep walking (25), and in sleep disorders in adults (26-29). Our results agreed with other authors (30) that the CAP rate can be a good measure of the SWS instability in children.

According to Parrino et al. (31), the CAP-A2 and -A3 subtypes are associated with sleep fragmentation in adults. Our data showed an increase in CAP-A2 in children with JIA that can indicate CAP-A2 subtype as a sensitive marker of sleep instability in JIA. CAP-A1 subtypes were less prominent than in controls $(P<0.01)$, another indication of sleep disturbance in JIA patients. The CAP phase A1 subtype as a marker of sleep stability in children has been questioned and described as having a higher rate in SWS than in other stages of NREM sleep $(23,24)$.

Pain has been reported as an important factor leading to changes in sleep patterns. The alpha EEG expression in pain syndromes during sleep stages has been reported as being increased in adults (32) and may not be different than alpha expression during wakefulness (33). Our results showed an increase in CAP-A2 subtypes during stage 3 of NREM sleep and A2 subtype definition includes alpha EEG that can be a good marker of sleep disruption in our subjects.

In our results, there was an abnormality of SWS, with a significant increase in all CAP parameters $(P<0.01)$ compared to matched controls, which is not identified by sleep stage scoring. This abnormality of SWS recognized by CAP may explain some of the physical changes noted in JIA, more particularly, changes in growth and body development (34). We acknowledge that our study did not perform any hormonal measurement, but it is well known that growth hormone secretion occurs predominantly during SWS (35) and that sleep fragmentation in these patients with chronic arthritis may affect this secretion. It is also known that adults have a consolidation of sleep with hypnotic drugs, and it can be beneficial as well as can decrease discomfort in patients with rheumatoid arthritis. CAP analyses suggest more sleep disturbances than conventional sleep analyses in children with JIA. The CAP scoring can be useful to investigate the relationship between chronic pain and SWS disruption.

\section{References}

1. Affleck G, Urrows $S$, Tennen $H$, Higgins $P$, Abeles M. Sequential daily relations of sleep, pain intensity, and attention to pain among women with fibromyalgia. Pain 1996; 68 : 363-368.
2. Ancoli-Israel S, Benca RM, Edinger JD, Krystal AD, Mendelson W, Moldofsky $\mathrm{H}$, et al. Panel discussion: changing how we think about insomnia. J Clin Psychiatry 2004; 65 (Suppl 8): 44-46. 
3. Ohayon MM. Specific characteristics of the pain/depression association in the general population. J Clin Psychiatry 2004; 65 (Suppl 12): 5-9.

4. Drewes AM. Pain and sleep disturbances with special reference to fibromyalgia and rheumatoid arthritis. Rheumatology 1999; 38: 1035-1038.

5. Cakirbay H, Bilici M, Kavakci O, Cebi A, Guler M, Tan U. Sleep quality and immune functions in rheumatoid arthritis patients with and without major depression. Int $J$ Neurosci 2004; 114: 245-256.

6. Mahowald MW, Mahowald ML, Bundlie SR, Ytterberg SR. Sleep fragmentation in rheumatoid arthritis. Arthritis Rheum 1989; 32: 974-983.

7. Hirsch M, Carlander B, Verge M, Tafti M, Anaya JM, Billiard $M$, et al. Objective and subjective sleep disturbances in patients with rheumatoid arthritis. A reappraisal. Arthritis Rheum 1994; 37: 41-49.

8. Rains JC, Penzien DB. Sleep and chronic pain: challenges to the alpha-EEG sleep pattern as a pain specific sleep anomaly. J Psychosom Res 2003; 54: 77-83.

9. Hauri P, Hawkins DR. Alpha-delta sleep. Electroencephalogr Clin Neurophysiol 1973; 34: 233-237.

10. Moldofsky H, Scarisbrick $P$, England R, Smythe H. Musculoskeletal symptoms and non-REM sleep disturbance in patients with "fibrositis syndrome" and healthy subjects. Psychosom Med 1975; 37: 341-351.

11. Roizenblatt $\mathrm{S}$, Moldofsky $\mathrm{H}$, Benedito-Silva AA, Tufik S. Alpha sleep characteristics in fibromyalgia. Arthritis Rheum 2001; 44: 222-230.

12. Moldofsky H, Lue FA, Smythe HA. Alpha EEG sleep and morning symptoms in rheumatoid arthritis. $J$ Rheumatol 1983; 10: 373-379.

13. Moldofsky $H$, Saskin $P$, Lue FA. Sleep and symptoms in fibrositis syndrome after a febrile illness. J Rheumatol 1988; 15: 1701-1704.

14. Carette S, Oakson G, Guimont C, Steriade M. Sleep electroencephalography and the clinical response to amitriptyline in patients with fibromyalgia. Arthritis Rheum 1995; 38: 1211-1217.

15. Mahowald ML, Mahowald MW. Nighttime sleep and daytime functioning (sleepiness and fatigue) in less well-defined chronic rheumatic diseases with particular reference to the 'alpha-delta NREM sleep anomaly'. Sleep Med 2000; 1: 195-207.

16. Harman K, Pivik RT, D'Eon JL, Wilson KG, Swenson JR, Matsunaga L. Sleep in depressed and nondepressed participants with chronic low back pain: electroencephalographic and behaviour findings. Sleep 2002; 25: 775-783.

17. Stores G, Crawford C. Arousal norms for children age 5-16 years based on home polysomnography. Technol Health Care 2000; 8: 285-290

18. Zamir G, Press J, Tal A, Tarasiuk A. Sleep fragmentation in children with juvenile rheumatoid arthritis. J Rheumatol 1998; 25: 1191-1197.

19. Labyak SE, Bourguignon C, Docherty S. Sleep quality in children with juvenile rheumatoid arthritis. Holist Nurs Pract
2003; 17: 193-200.

20. Rechtschaffen A, Kales A. Manual of standardized terminology: Techniques and scoring system for sleep stages of human subjects. Los Angeles: UCLA Brain Information Service/Brain Research Institute; 1968.

21. EEG arousals: scoring rules and examples: a preliminary report from the Sleep Disorders Atlas Task Force of the American Sleep Disorders Association. Sleep 1992; 15: 173-184.

22. Terzano MG, Parrino L, Smerieri A, Chervin R, Chokroverty $\mathrm{S}$, Guilleminault $\mathrm{C}$, et al. Atlas, rules, and recording techniques for the scoring of cyclic alternating pattern (CAP) in human sleep. Sleep Med 2002; 3: 187-199.

23. Lopes MC, Guilleminault C. Chronic snoring and sleep in children: a demonstration of sleep disruption. Pediatrics 2006; 118: e741-e746.

24. Lopes MC, Rosa A, Roizenblatt S, Guilleminault C, Passarelli C, Tufik $S$, et al. Cyclic alternating pattern in peripubertal children. Sleep 2005; 28: 215-219.

25. Guilleminault $\mathrm{C}$, Lee $\mathrm{JH}$, Chan $\mathrm{A}$, Lopes MC, Huang YS, da Rosa A. Non-REM-sleep instability in recurrent sleepwalking in pre-pubertal children. Sleep Med 2005; 6: 515-521.

26. Terzano MG, Parrino L, Boselli M, Spaggiari MC, Di Giovanni G. Polysomnographic analysis of arousal responses in obstructive sleep apnea syndrome by means of the cyclic alternating pattern. J Clin Neurophysiol 1996; 13: 145-155.

27. Terzano MG, Parrino L, Spaggiari MC, Palomba V, Rossi M, Smerieri A. CAP variables and arousals as sleep electroencephalogram markers for primary insomnia. Clin Neurophysiol 2003; 114: 1715-1723.

28. Zucconi M, Oldani A, Ferini-Strambi L, Smirne S. Arousal fluctuations in non-rapid eye movement parasomnias: the role of cyclic alternating pattern as a measure of sleep instability. J Clin Neurophysiol 1995; 12: 147-154.

29. Terzano MG, Mancia D, Salati MR, Costani G, Decembrino A, Parrino $\mathrm{L}$. The cyclic alternating pattern as a physiologic component of normal NREM sleep. Sleep 1985; 8: 137-145.

30. Bruni O, Ferri R, Novelli L, Finotti E, Miano S, Guilleminault C. NREM sleep instability in children with sleep terrors: the role of slow wave activity interruptions. Clin Neurophysiol 2008; 119: 985-992.

31. Parrino L, Smerieri A, Rossi M, Terzano MG. Relationship of slow and rapid EEG components of CAP to ASDA arousals in normal sleep. Sleep 2001; 24: 881-885.

32. Horne JA, Shackell BS. Alpha-like EEG activity in non-REM sleep and the fibromyalgia (fibrositis) syndrome. Electroencephalogr Clin Neurophysiol 1991; 79: 271-276.

33. Pilowsky I, Crettenden I, Townley M. Sleep disturbance in pain clinic patients. Pain 1985; 23: 27-33.

34. Luboshitzky R. Endocrine activity during sleep. J Pediatr Endocrinol Metab 2000; 13: 13-20.

35. Born J, Muth S, Fehm HL. The significance of sleep onset and slow wave sleep for nocturnal release of growth hormone (GH) and cortisol. Psychoneuroendocrinology 1988; 13: $233-243$ 